\title{
DNCON2_Inter: Predicting interchain contacts for homodimeric and homomultimeric protein complexes using multiple sequence alignments of monomers and deep learning
}

\section{Farhan Quadir}

University of Missouri

Raj Roy

University of Missouri

\section{Randal Halfmann}

Stowers Institute for Medical Research

Jianlin Cheng ( $\nabla$ chengji@missouri.edu )

University of Missouri

\section{Research Article}

Keywords: inter-protein contact, interchain contact, intrachain contact, intra-protein contact, protein complex structure, residueresidue contact, homodimer, homomultimer, homo-oligomer, deep learning, contact prediction, quaternary structure, coevolution, interface contact

Posted Date: February 16th, 2021

DOI: https://doi.org/10.21203/rs.3.rs-228041/v1

License: () (1) This work is licensed under a Creative Commons Attribution 4.0 International License. Read Full License 


\section{Abstract}

Deep learning methods that achieved great success in predicting intrachain residue-residue contacts have been applied to predict interchain contacts between proteins. However, these methods require multiple sequence alignments (MSAs) of a pair of interacting proteins (dimers) as input, which are often difficult to obtain because there are not many known protein complexes available to generate MSAs of sufficient depth for a pair of proteins. In recognizing that multiple sequence alignments of a monomer that forms homomultimers contain the co-evolutionary signals of both intrachain and interchain residue pairs in contact, we applied DNCON2 (a deep learning-based protein intrachain residue-residue contact predictor) to predict both intrachain and interchain contacts for homomultimers using multiple sequence alignment (MSA) and other coevolutionary features of a single monomer followed by discrimination of interchain and intrachain contacts according to the tertiary structure of the monomer. We name this tool DNCON2_Inter. Allowing true-positive predictions within two residue shifts, the best average precision was obtained for the Top-L/10 predictions of $22.9 \%$ for homodimers, and $17.0 \%$ for higher order homomultimers. In some instances, especially where interchain contact densities are high, DNCON2_Inter predicted interchain contacts with $100 \%$ precision. We also developed Con_Complex, a complex structure reconstruction tool that uses predicted contacts to produce the structure of the complex. Using Con_Complex, we show that the predicted contacts can be used to accurately construct the structure of some complexes. Our experiment demonstrates that monomeric multiple sequence alignments can be used with deep learning to predict interchain contacts of homomeric proteins.

\section{Introduction}

Proteins are one of the most important and heavily studied biological molecules. While most proteins form individual threedimensional structures, they tend to interact with each other to gain functional properties. In fact, most proteins are symmetrical oligomeric complexes with two or more subunits ${ }^{1}$, and approximately two-thirds of human enzymes are homooligomers ${ }^{2}$.

Since the functionality of most proteins heavily depends on oligomerization, and wet laboratory experiments with actual proteins are time-consuming and expensive, there is a need to develop accurate computational tools to make such predictions quickly. Machine learning methods have been developed to facilitate computational modeling of both protein tertiary structures and quaternary structures. However, recent focus has been on the development of computational tools for predicting intrachain (within the same chain) residue-residue contacts and distances to guide tertiary structure modeling ${ }^{3,4}$. Some of these methods have performed well in the 12th and 13th Critical Assessment of Techniques for Protein Structure Prediction (CASP) competitions ${ }^{5-14}$.

Unlike tertiary structure modeling, most of the tools on protein complexes are developed to classify whether two proteins are interacting or not, with some tools developed exclusively for docking purposes. Only a small number of tools were developed to predict interchain contacts leveraging interchain residue-residue co-evolutionary signals embedded in the MSA of a pair of interacting protein homologues (i.e., interlogs) ${ }^{3,4,15}$. Relevant to the present work Zhou et al. ${ }^{4}$, and Zeng et al. ${ }^{16}$ predicted interchain contacts using a deep learning-based intrachain contact prediction tool (RaptorX-ComplexContact) without training the system for interchain contact prediction. Their work involved generating MSAs using homology-based, phylogeny-based, and genome-based interlog searches, which is similar to the methods employed by Baker ${ }^{15}$ and Marks ${ }^{3}$. Baker ${ }^{15}$ used a $^{2}$ pseudo-likelihood-based covariance approach to predict interprotein contacts in bacterial proteins. The method involved the computation of residue-residue coupling strength between all the interacting protein pairs in the MSA based on the GREMLIN model. The coupling strengths were then ranked and used to compute a score, which was used to derive the distance restraints. EVcomplex ${ }^{3}$ used evolutionary couplings $(E C)$ to predict the interface contacts between prokaryotic proteins. Applying EVcouplings ${ }^{17}$ to the paired MSA and using the pseudo-likelihood maximization (PLM) approach, both inter-EC and intra-EC were obtained. The normalized raw reliability score (EVcomplex score) was calculated using the inter-EC portion. The interchain residue pairs were ranked according to the EVcomplex scores, and pairs with scores $\geq 0.8$ were considered to be in contact with high confidence. 
The works above focused on determining the heteromeric interprotein contacts and require MSAs of homologous interlogs as input. However, obtaining MSAs of interlogs of sufficient depth is difficult because there are fewer known protein complexes than known monomers. However, the situation is different for homo-oligomers because their units are the same monomer. Therefore, both intrachain and interchain residue-residue co-evolutionary signals exist within the same MSA of the monomer. That is, both the co-evolution signal between residues $\mathrm{i}$ and $\mathrm{j}$ within a monomer, and that between residue $\mathrm{i}$ in the monomer and residue $\mathrm{j}$ in its identical partner are mixed in the MSA of the monomer. This phenomenon has been recognized before but has not been leveraged to predict interchain contacts in homo-oligomers ${ }^{18}$.

The objective of this study focuses on predicting the interprotein contact of homomeric proteins (chains having similar amino acid sequences) using MSAs of the monomer unit in homo-oligomers. Similar to Zeng et al. ${ }^{16}$, and Zhou et al. ${ }^{4}$, our approach can be applied to both prokaryotic and eukaryotic proteins wherever sufficiently deep MSAs are available. Unlike the above approach to generate MSAs, ours directly extracts co-evolutionary features of homomeric proteins from homology-based MSAs constructed without any special genome- and phylogeny-based methods. We then directly apply DNCON2 ${ }^{5}$, a deep learning method trained to predict intrachain contacts, to these features to predict both intrachain and interchain contacts, which we distinguish according to the tertiary structures of monomers (See Supplementary Section Figure S1 for the DNCON2 deep learning architecture, and Table S1 for the list of features used by DNCON2). This paper focuses on proving this concept, while future work will focus on developing an exclusive deep-learning-based interprotein contact predictor. Since this development is based on DNCON2, we name our method DNCON2_Inter.

Interchain protein contact predictions are not only useful to identify protein-protein interactions, but also in the construction of complex structures. Some docking tools use interchain contacts as additional restraints to compute better quaternary structures. Since more accurate interprotein contacts lead to better quality quaternary structures, this research will pave the way to improve de novo interprotein contact prediction and complex structure construction methods.

\section{Methods}

\section{Dataset preparation}

The list of homo-oligomers was obtained from the 3DComplex dataset ${ }^{19}$. We selected our protein dataset after the Protein Data Bank (PDB) files underwent a cascade of filtration steps. We discarded the proteins that had interactions with nucleic acids and cleaned PDB files according to the process described in Figure 1.

Since PDB files tend to contain more information than necessary, we cleaned them using MULTICOM Toolbox ${ }^{14,20}$ as shown in Figure 1. DSSP ${ }^{21}$ is applied to generate secondary structure and solvent accessibility information for each PDB file. If DSSP can be generated for a protein, the entire PDB file is split into individual files corresponding to the chains present, keeping only the ATOM $(x, y$, and $z$ coordinate) portion. The residue numbers are then reindexed to ensure every residue number begins with a ' 1 ' and continues without any breaks. If the FASTA sequence similarity between chains is less than $95 \%$, we discard the protein. The individual chain-wise files are further processed to remove the mismatched residue information, and residue numbers were reindexed if necessary, for homogenizing the residue similarity between chains.

Proteins with more than $30 \%$ sequence identity were removed using the "mmseqs" 22 . We also excluded the proteins which did not have any interchain contact (distance between interchain residues was $>6.0 \AA$ ). Several proteins that failed with FreeContact ${ }^{23}$, PSICOV ${ }^{24}$, and PSI-BLAST ${ }^{25}$ in the feature generation process of DNCON2 were also excluded. Finally, we obtained a dataset of 8681 homodimeric proteins and 6764 higher order homo-oligomeric (hereafter, "homomultimeric") proteins for prediction and analysis. The homodimeric and homomultimeric datasets did not overlap and were treated separately.

\section{Prediction and Evaluation of interchain contacts}


Since dimeric proteins contain two chains, the underlying process is relatively simple. Hence, in this study, we only describe predicting the homomultimeric proteins' interchain contacts, which implicitly covers the dimers. As described in Figure 2, the cleaned chain-wise split PDBs and FASTA sequences were given input to our prediction and evaluation system. The coordinates of chain ' $A$ ' (the first chain) were used to compute the intrachain contacts. An intrachain contact between two residues $i$ and $j$ is said to exist if the Euclidean distance between the respective $C_{\boldsymbol{\beta}}$ ( $C_{\boldsymbol{\alpha}}$ for glycine) atoms of residues $i$ and $j$ is less than or equal to $8.0 \AA^{5,14}$. If the $C_{\boldsymbol{\beta}}$ was unavailable, we chose $C_{\boldsymbol{\alpha}}$ instead. We also defined interchain contact between chains in a protein if the Euclidean distance between the heavy atoms of the residues in the respective chains is less than or equal to $6.0 \AA^{3,4,15}$. We obtained a pairwise contact list between the first chain (chain $A$ ) and the other chains. For example, if a protein contains four chains $A, B, C$, and $D$, we pair them as $A B, A C$, and $A D$ and determine the interchain contacts. From this distribution, we select the pair with the highest number of contacts and base the rest of our analysis on this.

To predict the interchain contacts, the FASTA sequence of the first chain (i.e., chain A) was fed into our predictor programDNCON2 - which outputs the predicted intrachain contact map. This predicted contact map was processed to filter out the short-range contacts (contacts whose sequence separation is less than 6). The interchain contacts are obtained as follows:

1. We removed the matching true intrachain contacts from the prediction. Our assumption states that the remaining contacts in the prediction contact map should correspond to some sort of interchain contact.

2. We then computed the precision using the contact map obtained from (1) and the true interchain contacts using InterConEva- an extension of ConEva ${ }^{26}$ tool for interchain contact evaluation. It should be noted that DNCON2 outputs the upper triangle of the prediction matrix since the intrachain contact map is symmetric. However, in interchain prediction, the entire matrix needs to be considered since contact between residues $i$ and $j$ of two different chains is different from contact between residue $j$ and $i$. If the final predicted contact map had a contact $(x, y)$, we checked for both $(x, y)$ and $(y, x)$ in the true interchain contact map and considered them to be two separate contacts.

We experimented further by applying relaxations to the above parts (1) and (2). We termed them as "relax removal," and " relaxation," respectively. During relax removal, we removed the true intrachain contacts from the predicted contact map as follows:

1. If position (i,j) is a true intrachain contact, and the relax parameter is $n$ (where, $n=0,1$, and 2 ), then let $X=[i-n, i+n]$ and $Y=$ $[j-n, j+n]$

2. Remove all contacts $\left(X_{p}, Y_{q}\right)$ from the predicted contact map where $X_{p}=\{i-n, i-n+1, \ldots, i+n\}$ and $Y_{q}=\{j-n, j-n+1, \ldots, j+n\}$. This removes (sets to zero) a square matrix of dimension $n \times n$ centered at $(\mathrm{i}, \mathrm{j})$ from the predicted intrachain contact map.

Relaxation follows a similar approach. If a prediction is found for the position $(i, j)$, we look for contacts within the square matrix of dimension $n \times n$ centered at $(\mathrm{i}, \mathrm{j})$ in the true interchain contact map. If any nonzero value is found within the $\mathrm{n} \times \mathrm{n}$ square region centered at $(\mathrm{i}, \mathrm{j})$ of the true interchain contacts, it is counted as a successfully predicted contact. The value of $n$ is ranged from 0 to 2 since we perform relaxation within two residue shifts.

We also selected one best-case result and reconstructed the complex structure using our recently developed tool Con_Complex, which can reconstruct the quaternary structure of multimers by leveraging the simulated annealing protocol of CNS (Crystallography and NMR System) ${ }^{27,28}$. It uses the monomer PDB file and the predicted inter-protein contacts to reconstruct the homomultimeric complex structure. We compare interchain contact maps using InterConEva, visualize quaternary structures using Chimera ${ }^{29}$, and calculate TMscore using TM-Align ${ }^{30}$. Additionally, we randomly selected 25 homodimer proteins with non-zero precisions from our experiment, and compared our results to the predictions made by RaptorXComplexContact webserver.

\section{Results And Discussions}

The Precision of Interchain Contact Prediction

Page 4/13 
The results from Figure 3 (Supplementary Section Figure S3 is a line graph of Figure $\mathbf{3}$ ) shows that as we perform relax removal and relaxation, the interchain precision increases. We obtain a maximum average interchain precision of $22.9 \%$ and $17.0 \%$ for homodimers and homomultimers, respectively, for the Top-L/10 group with relax removal = 2 and relaxation $=2$. When compared with the precision results obtained from random predictions (Supplementary Section Tables S3 and S4), for the Top-L/10 with relax removal $=2$ and relaxation $=2$, our prediction is 4.1 times greater for homodimers and 3.5 times better for homomultimers. For all groups, increasing relaxation tends to bring about slight increases in precision values. This increase is due to the consideration of true-positive hits over a flexible boundary that enables the system to discover more contacts within a fixed vicinity. However, from Top-L/5 and beyond, precision drops drastically for all the graphs since we are dividing by a bigger number.

In further analysis, we compare precision against contact density. Contact density is the total number of native contacts per length of the protein ${ }^{4}$. We discuss the contact density and precision of the Top-2L predictions of homodimers without any relax removal. Figure 4 shows that precision increases with increasing contact density for the Top-2L group. We observe that performing relaxation from 0 to 1 increases the precision for contact densities of all ranges. In this case, precision almost doubles when contact densities are less than 3.50, while, for contact densities beyond 3.50, the precision increases slightly. Performing relaxation from 1 to 2 increases precision but lesser than what is observed when relaxing from 0 to 1 . Relaxation from 1 to 2 affects precision values less for contact densities $\geq 3.50$. Proteins with high contact density get predicted better than proteins with low contact density. It should be noted that most proteins have very sparse interchain contacts and thus have low contact densities. Also, the number of proteins with high contact densities is significantly less (See Supplementary Section Table S2), so these proteins play little role in the overall precision. (Detailed explanation of how relax removal and relaxation effects the precision is described in Supplementary Section 10.)

\section{Case study of one of the best predictions}

We investigate the results of one of the best predictions PDB code: 1A64. Details are available in the Supplementary Section 7 (Tables S6, and Figures S4 and S5, respectively)

Although DNCON2 predicted intrachain contacts for this target with relatively low precision, all the interchain contacts were correctly predicted (Table S7 Supplementary Section), which is also confirmed visually by the bulk overlapping of the green and red dots, as seen in Figure 5 (a) and Figure S4 (Supplementary Section). As we perform relax removal (A to B to C), the mispredicted green contacts in the contact maps become sparse due to removing the false-positive (assumed to be intrachain) contacts. The green dots that overlap with the red dots are correct inter-chain contact predictions. Using these contact maps, we use Con_Complex to create quaternary structures and visualize them using Chimera ${ }^{29}$ (Figure 5 (b) and

Supplementary Section Figure S5). Finally, we evaluate the resulting structures' accuracy using TMAlign ${ }^{30}$. Our results, in Figure 5 (c), indicate very close resemblance to the actual structures with almost perfect TM-scores for the relax removed contacts since intrachain contacts are almost eliminated, leaving behind only true-positive interchain contacts. (Results for another case study on the protein 1IHR can be found in Supplementary Section 8.0.)

\section{Comparison between DNCON2_Inter and RaptorX-ComplexContact}

In our final analysis, we randomly selected 25 homodimer targets which had non-zero precisions from our interchain contact prediction mechanism. We submitted these targets for interchain contact prediction to the RaptorX-ComplexContact webserver. In Figure 6 we only show the results for relaxation $=2$ since ComplexContact showed the best results at this threshold. The detailed results at different relaxations are reported in Supplementary Section Table S5 and Figure S2.

It is evident from Figure 6 that, for the selected homodimers, DNCON2_Inter outperforms ComplexContact by almost four times for the Top-10 selected contacts. Since ComplexContact bases its MSA on genome-based and phylogeny-based interlogs, it is more suited for heteromeric contact prediction. So we apply a similar interchain contact prediction method as described for 
DNCON2_Inter. Since ComplexContact also outputs the intrachain contact map, from this, we removed the true intrachain contacts and re-evaluated the results. Table 1 shows the best result obtained by ComplexContact for relax removal $=1$ and relaxation $=2$, in comparison to that obtained using DNCON2_Inter (Details in Supplementary Section Table S6). Although we see improvements in the precision of ComplexContact as compared to what is seen in Table S5, DNCON2_Inter is still 1.5 times better than ComplexContact for the Top-L/10 precisions.

Table 1: Comparison between the best precisions (\%) of interchain contacts predicted by ComplexContact, and DNCON2_Inter on 25 random non-zero precision proteins sampled from the homodimer dataset after removing the true contacts from the predicted intrachain contacts. The best precision for ComplexContact is obtained for relax removal $=1$ and relaxation $=2$ :

\begin{tabular}{|c|c|c|c|c|c|c|c|c|c|c|c|c|c|}
\hline \multicolumn{7}{|c|}{ ComplexContact_Intra - True_intrachain_contacts } & \multicolumn{7}{|c|}{ DNCON2_Inter } \\
\hline Top-5 & $\begin{array}{l}\text { Top- } \\
10\end{array}$ & $\begin{array}{l}\text { Top- } \\
\text { L/10 }\end{array}$ & $\begin{array}{l}\text { Top- } \\
\text { L/5 }\end{array}$ & $\begin{array}{l}\text { Top- } \\
\text { L/2 }\end{array}$ & $\begin{array}{l}\text { Top- } \\
\text { L }\end{array}$ & $\begin{array}{l}\text { Top- } \\
2 \mathrm{~L}\end{array}$ & $\begin{array}{l}\text { Top- } \\
5\end{array}$ & $\begin{array}{l}\text { Top- } \\
10\end{array}$ & $\begin{array}{l}\text { Top- } \\
\text { L/10 }\end{array}$ & $\begin{array}{l}\text { Top- } \\
\text { L/5 }\end{array}$ & $\begin{array}{l}\text { Top- } \\
\text { L/2 }\end{array}$ & $\begin{array}{l}\text { Top- } \\
\text { L }\end{array}$ & $\begin{array}{l}\text { Top- } \\
2 \mathrm{~L}\end{array}$ \\
\hline 44.80 & 46.00 & 52.49 & 47.64 & 35.26 & 21.93 & 11.06 & 79.2 & 81.60 & 78.49 & 72.14 & 54.50 & 34.34 & 17.45 \\
\hline
\end{tabular}

\section{Conclusion, Limitation, And Future Work}

This study demonstrates that DNCON2, a deep learning-based intrachain contact predictor, can successfully be used to predict interchain residue-residue contacts for homodimeric and homomultimeric protein complexes from the multiple sequence alignment of a monomer. Although the precision of the predictor is not high on average due to it being trained mainly for intrachain contact prediction, the prediction accuracy is still much higher than a random predictor. In some cases, our approach predicted more interchain contacts than intrachain contacts with very high precision. The results provide good evidence that deep learning tools can be used to train for such a task using co-evolutionary features obtained directly from homology-based MSA of a monomer. Unlike the previous works of Hopf et al. ${ }^{3}$, Ovchinnikov et al. ${ }^{15}$, Zhou et al. ${ }^{4}$, and Zeng et al. ${ }^{16}$, that requires hard-to-obtain MSAs of interlogs as input, our approach can be readily applied to any homodimers and homomultimers. Moreover, the quality of the interchain contacts directly influences complex structure construction. Also, removing false-positive contacts through relax removal can significantly increase the TM-score of the constructed 3D structures of the complexes. Our results conclude that relax removal $=2$ and relaxation $=2$ gives us the best precision, especially for Top-L/10 contacts.

Since DNCON2 has been trained for intrachain contact prediction, it has not reached the best performance at predicting interchain contacts. Thus, our results encourage us to train a complex deep learning architecture specific to predicting interchain contacts of homodimers and homomultimers in the future.

\section{Declarations}

\section{Acknowledgment}

Research reported in this publication was supported in part by two NSF grants (DBI 1759934 and IIS1763246) to JC, an NIH grant (R01GM093123), and two Department of Energy grants (DE-AR0001213 and DE-SC0021303) to JC.

\section{Author contributions}

J.C. conceived the project. J.C., F.Q., R.R. and R.H. designed the methods. F.Q. performed the major part of the experiment including dataset development, performing the experiment, evaluation, statistical analysis of results and generation of figures and tables. R.R. developed Con_Complex which was used to reconstruct the structure of the complexes. F.Q. and J.C. wrote the manuscript. All authors reviewed and edited the manuscript.

\section{Competing interests}


The authors declare no competing interests.

\section{References}

1. Goodsell, D. S. \& Olson, A. J. Structural symmetry and protein function. Annual Review of Biophysics and Biomolecular Structure vol. 29 105-153 (2000).

2. Matthews, J. M. \& Sunde, M. Dimers, oligomers, everywhere. Advances in Experimental Medicine and Biology vol. 747 118 (2012).

3. Hopf, T. A. et al. Sequence co-evolution gives 3D contacts and structures of protein complexes. eLife3, (2014).

4. Zhou, T.-M., Wang, S. \& Xu, J. Deep learning reveals many more inter-protein residue-residue contacts than direct coupling analysis. biorxiv.org10812 LNBI, 295-296 (2018).

5. Adhikari, B., Hou, J. \& Cheng, J. DNCON2: Improved protein contact prediction using two-level deep convolutional neural networks. Bioinformatics34, 1466-1472 (2018).

6. Alquraishi, M. \& Valencia, A. AlphaFold at CASP13. Bioinformatics35, 4862-4865 (2019).

7. Cheng, J. et al. Estimation of model accuracy in CASP13. Proteins: Structure, Function, and Bioinformatics87, 1361-1377 (2019).

8. Schaarschmidt, J., Monastyrskyy, B., Kryshtafovych, A. \& Bonvin, A. M. J. J. Assessment of contact predictions in CASP12: Co-evolution and deep learning coming of age. Proteins: Structure, Function, and Bioinformatics86, 51-66 (2018).

9. Senior, A. W. et al. Improved protein structure prediction using potentials from deep learning. Nature577, 706-710 (2020).

10. Shrestha, R. et al. Assessing the accuracy of contact predictions in CASP13. Proteins: Structure, Function, and Bioinformatics87, 1058-1068 (2019).

11. Wang, S., Sun, S., Li, Z., Zhang, R. \& Xu, J. Accurate De Novo Prediction of Protein Contact Map by Ultra-Deep Learning Model. PLOS Computational Biology13, e1005324 (2017).

12. Wang, S., Sun, S. \& Xu, J. Analysis of deep learning methods for blind protein contact prediction in CASP12. Proteins: Structure, Function, and Bioinformatics86, 67-77 (2018).

13. Xu, J. \& Wang, S. Analysis of distance-based protein structure prediction by deep learning in CASP13. Proteins: Structure, Function, and Bioinformatics87, 1069-1081 (2019).

14. Hou, J., Wu, T., Guo, Z., Quadir, F. \& Cheng, J. The MULTICOM Protein Structure Prediction Server Empowered by Deep Learning and Contact Distance Prediction. in Methods in Molecular Biology vol. 2165 13-26 (Humana Press Inc., 2020).

15. Ovchinnikov, S., Kamisetty, H. \& Baker, D. Robust and accurate prediction of residue-residue interactions across protein interfaces using evolutionary information. eLife3, (2014).

16. Zeng, H. et al. ComplexContact: A web server for inter-protein contact prediction using deep learning. Nucleic Acids Research46, W432-W437 (2018).

17. Hopf, T. A. et al. The EVcouplings Python framework for coevolutionary sequence analysis. Bioinformatics35, 1582-1584 (2019).

18. Uguzzoni, G. et al. Large-scale identification of coevolution signals across homo-oligomeric protein interfaces by direct coupling analysis. Proceedings of the National Academy of Sciences of the United States of America114, E2662-E2671 (2017).

19. Levy, E. D., Pereira-Leal, J. B., Chothia, C. \& Teichmann, S. A. 3D Complex: A Structural Classification of Protein Complexes. PLoS Computational Biology2, e155 (2006).

20. Cheng, J., Li, J., Wang, Z., Eickholt, J. \& Deng, X. The MULTICOM toolbox for protein structure prediction. BMC Bioinformatics13, 65 (2012).

21. Kabsch, W. \& Sander, C. Dictionary of protein secondary structure: Pattern recognition of hydrogen-bonded and geometrical features. Biopolymers22, 2577-2637 (1983). 
22. Hauser, M., Steinegger, M. \& Söding, J. MMseqs software suite for fast and deep clustering and searching of large protein sequence sets. Bioinformatics32, 1323-1330 (2016).

23. Kaján, L., Hopf, T. A., Kalaš, M., Marks, D. S. \& Rost, B. FreeContact: Fast and free software for protein contact prediction from residue co-evolution. BMC Bioinformatics15, 85 (2014).

24. Jones, D. T., Buchan, D. W. A., Cozzetto, D. \& Pontil, M. PSICOV: Precise structural contact prediction using sparse inverse covariance estimation on large multiple sequence alignments. Bioinformatics28, 184-190 (2012).

25. Altschul, S. F. et al. Gapped BLAST and PSI-BLAST: A new generation of protein database search programs. Nucleic Acids Research vol. 25 3389-3402 (1997).

26. Adhikari, B., Nowotny, J., Bhattacharya, D., Hou, J. \& Cheng, J. ConEVA: A toolbox for comprehensive assessment of protein contacts. BMC Bioinformatics17, 517 (2016).

27. Brunger, A. T. Version 1.2 of the crystallography and nmr system. Nature Protocols2, 2728-2733 (2007).

28. Brünger, A. T. et al. Crystallography \& NMR system: A new software suite for macromolecular structure determination. Acta Crystallographica Section D: Biological Crystallography54, 905-921 (1998).

29. Pettersen, E. F. et al. UCSF Chimera?A visualization system for exploratory research and analysis. Journal of Computational Chemistry25, 1605-1612 (2004).

30. Zhang, Y. \& Skolnick, J. TM-align: A protein structure alignment algorithm based on the TM-score. Nucleic Acids Research33, 2302-2309 (2005).

\section{Figures}

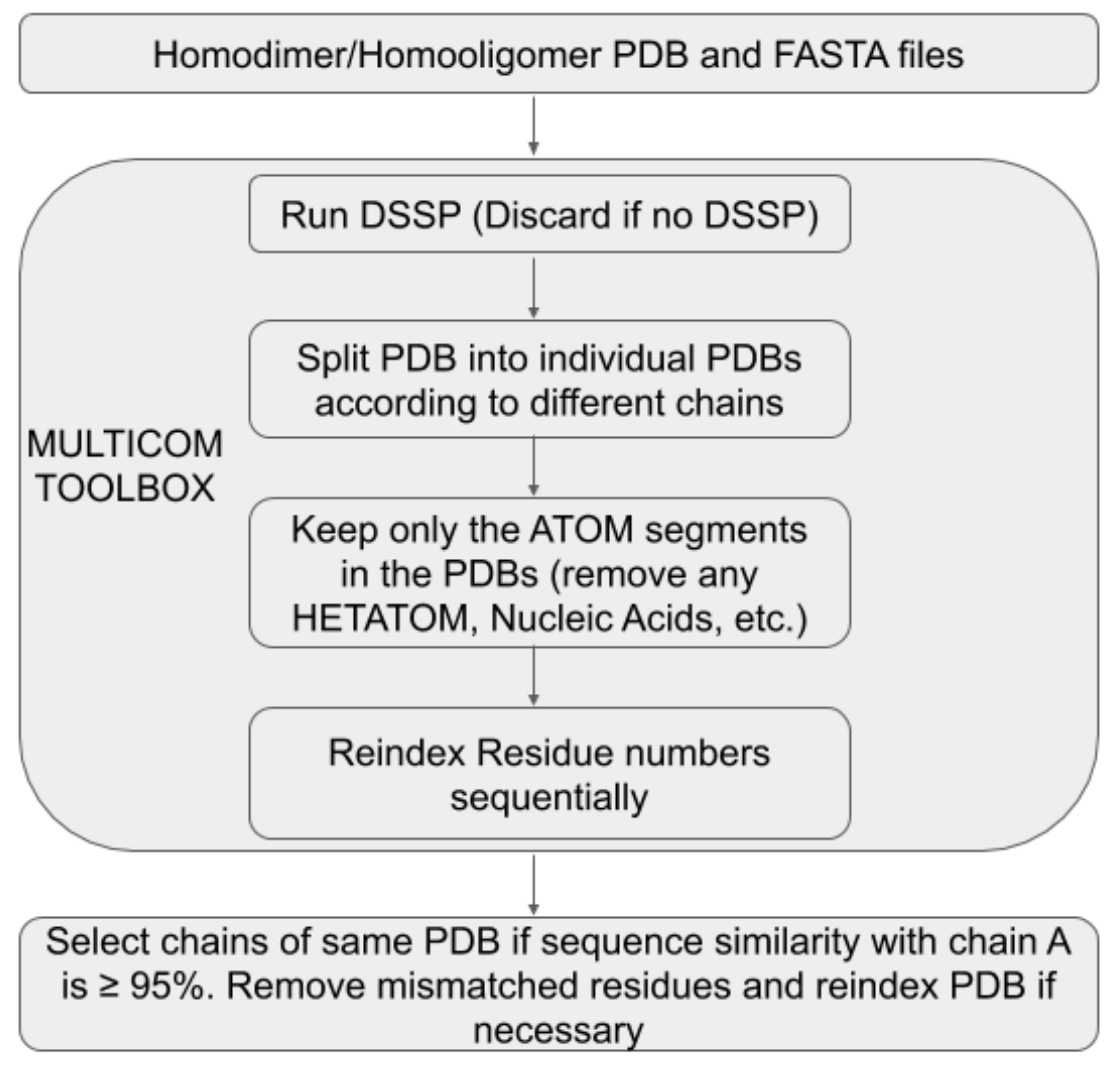

\section{Figure 1}

Diagram describing how the input PDB file was pre-processed using the MULTICOM TOOLBOX to clean up the PDB files. If no DSSP is available, the PDB was removed from our list. The individual chains in the multimer PDB were separated into individual files containing the ATOM ( $x, y$, and $z$ coordinates) segments only while discarding all other information. Only chain 
pairs whose FASTA sequences match $95 \%$ or more were kept, and any mismatched residues were removed to ensure homogeneity between chains.

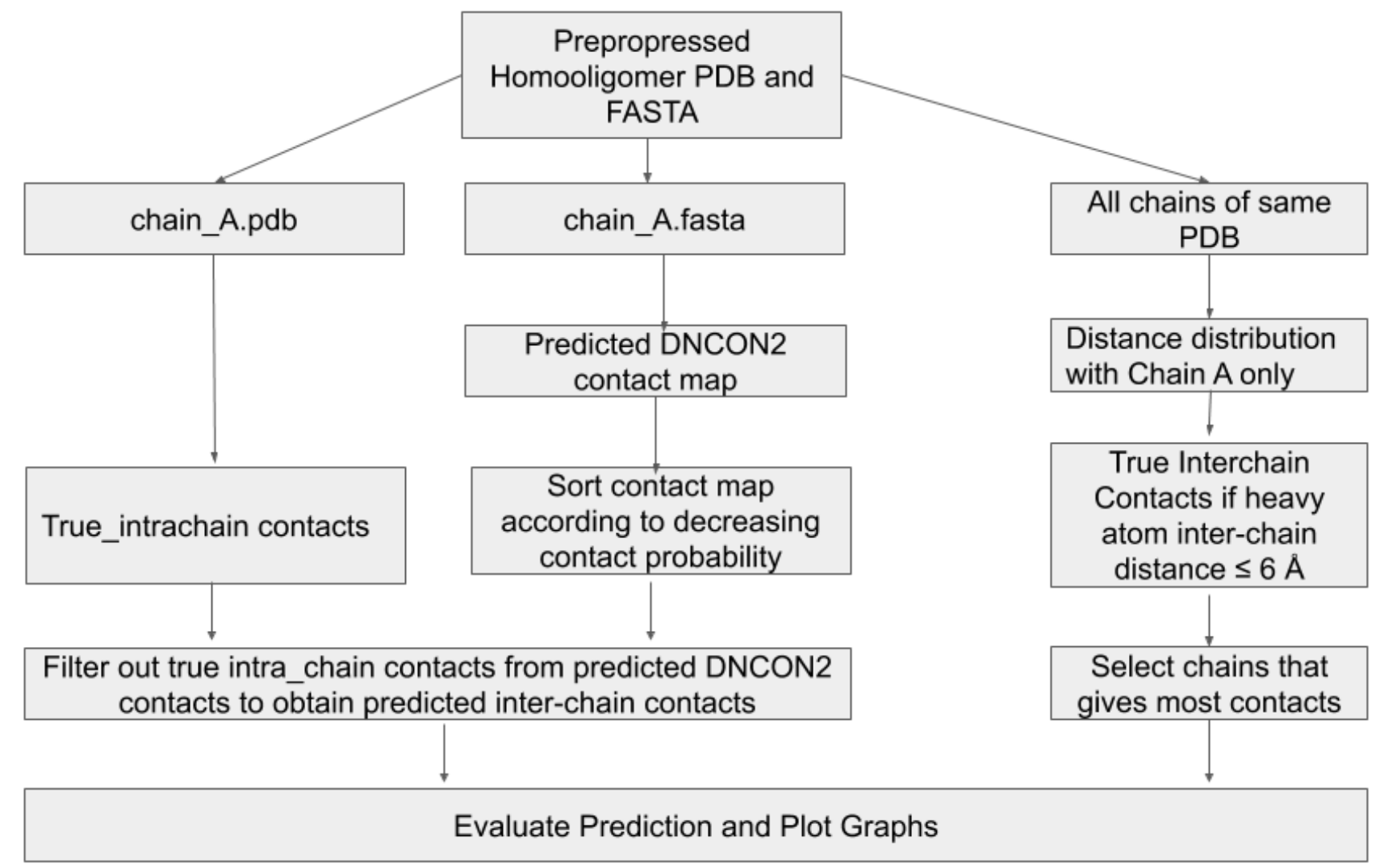

\section{Figure 2}

Workflow diagram describing how the pre-processed input PDB and FASTA sequence was used to derive true intrachain contacts, true interchain contacts, predicted interchain contacts, and finally, obtain the evaluation and visualization of the prediction. 
(a) Average precision for Inter-chain contact predictions of Homodimers

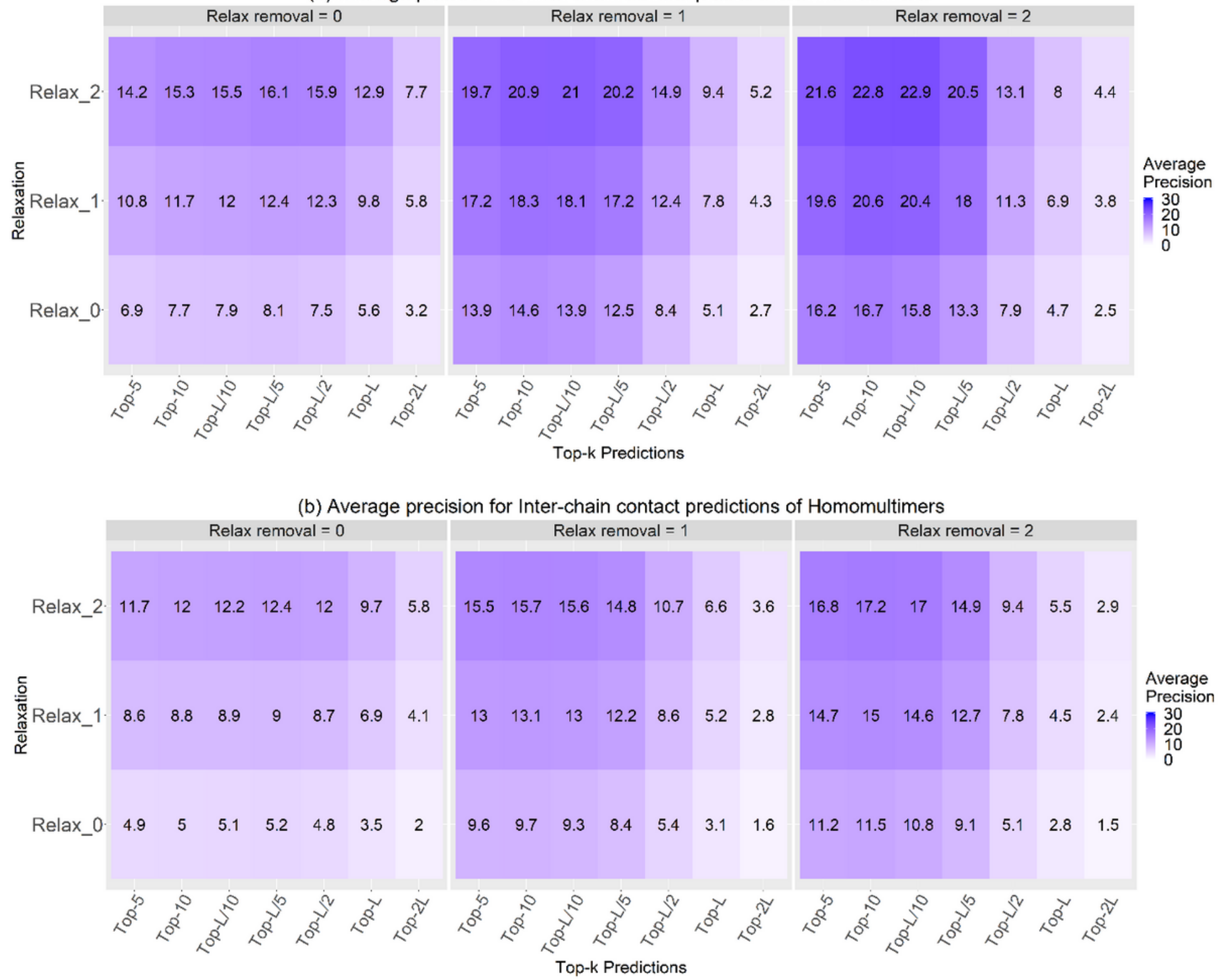

\section{Figure 3}

The precision heatmap of interchain contact predictions for the (a) homodimers and (b) homomultimers for the Top-k predictions where $k=5,10, L / 10, L / 5, L / 2, L$, and $2 \mathrm{~L}$. For all categories, as we do more relax removal and relaxation, precision values increase within the respective Top-k categories. Relax removal $=2$ and relaxation $=2$ shows the best precision of $22.9 \%$ for homodimers and $17.0 \%$ for homomultimers within the Top-L/10 predictions. 


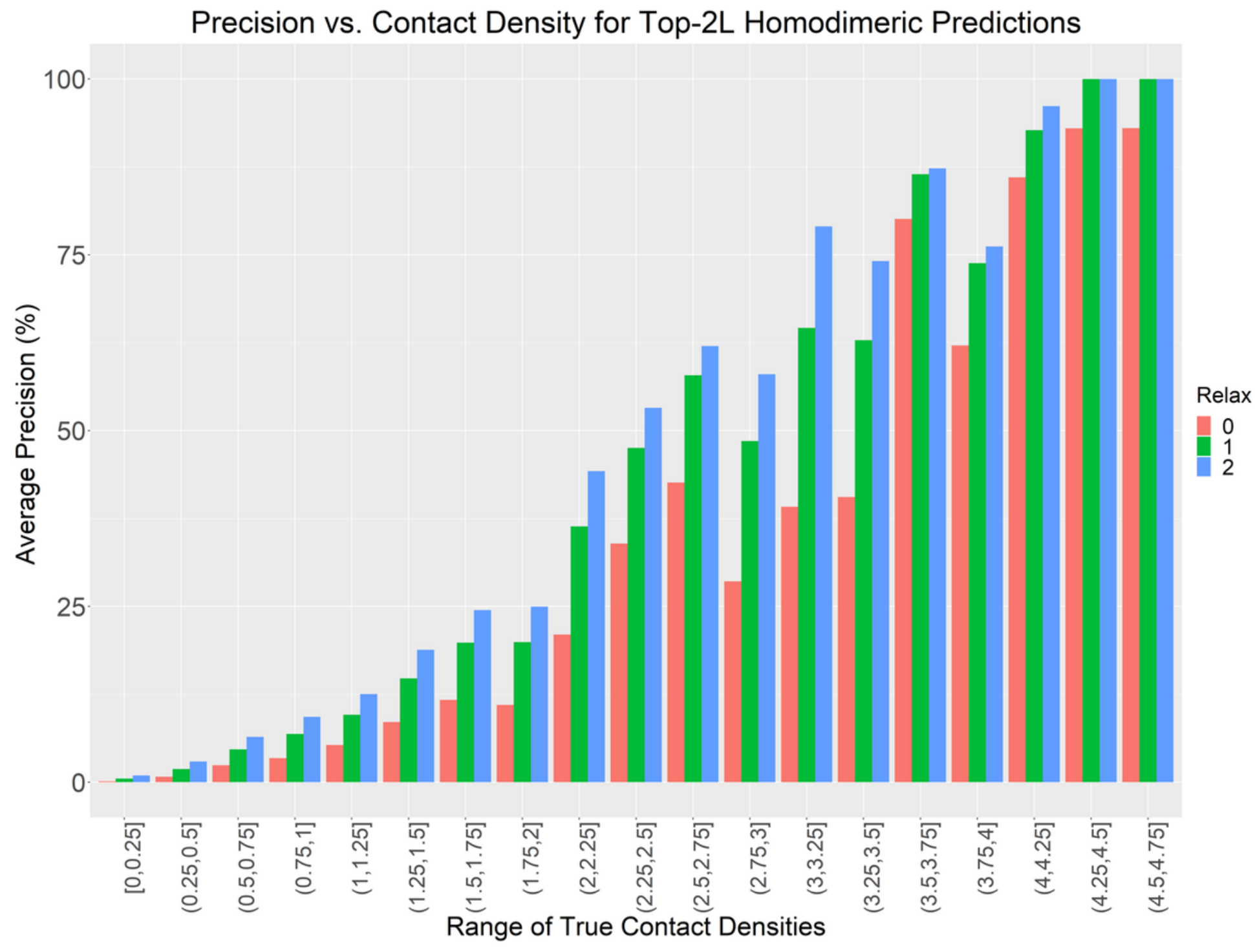

Figure 4

Bar plot depicting the prediction of Top $2 \mathrm{~L}$ interchain contact predictions changes as contact density varies with no relaxation removal. We can see that high contact density leads to high precision. Relaxation has little effect on precision when contact densities are beyond 3.50 . 
(a) Contact map

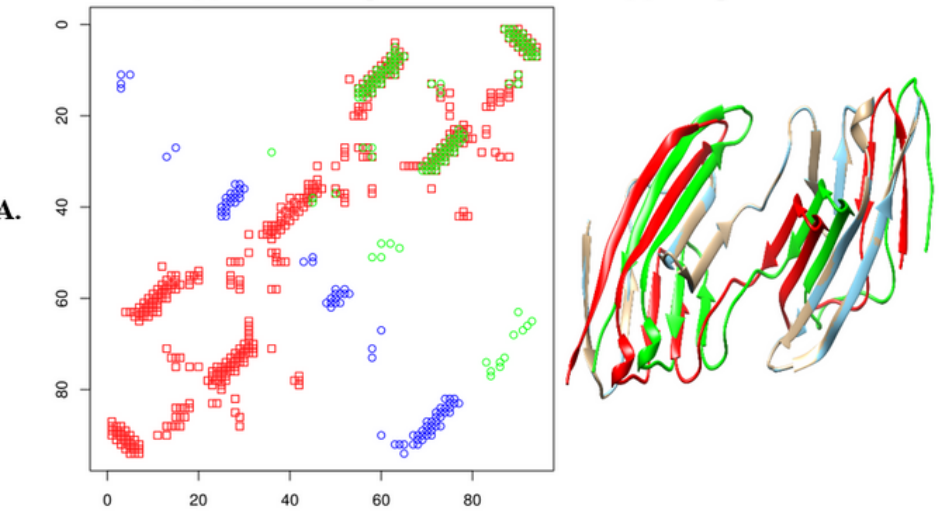

(c) Complex Quality
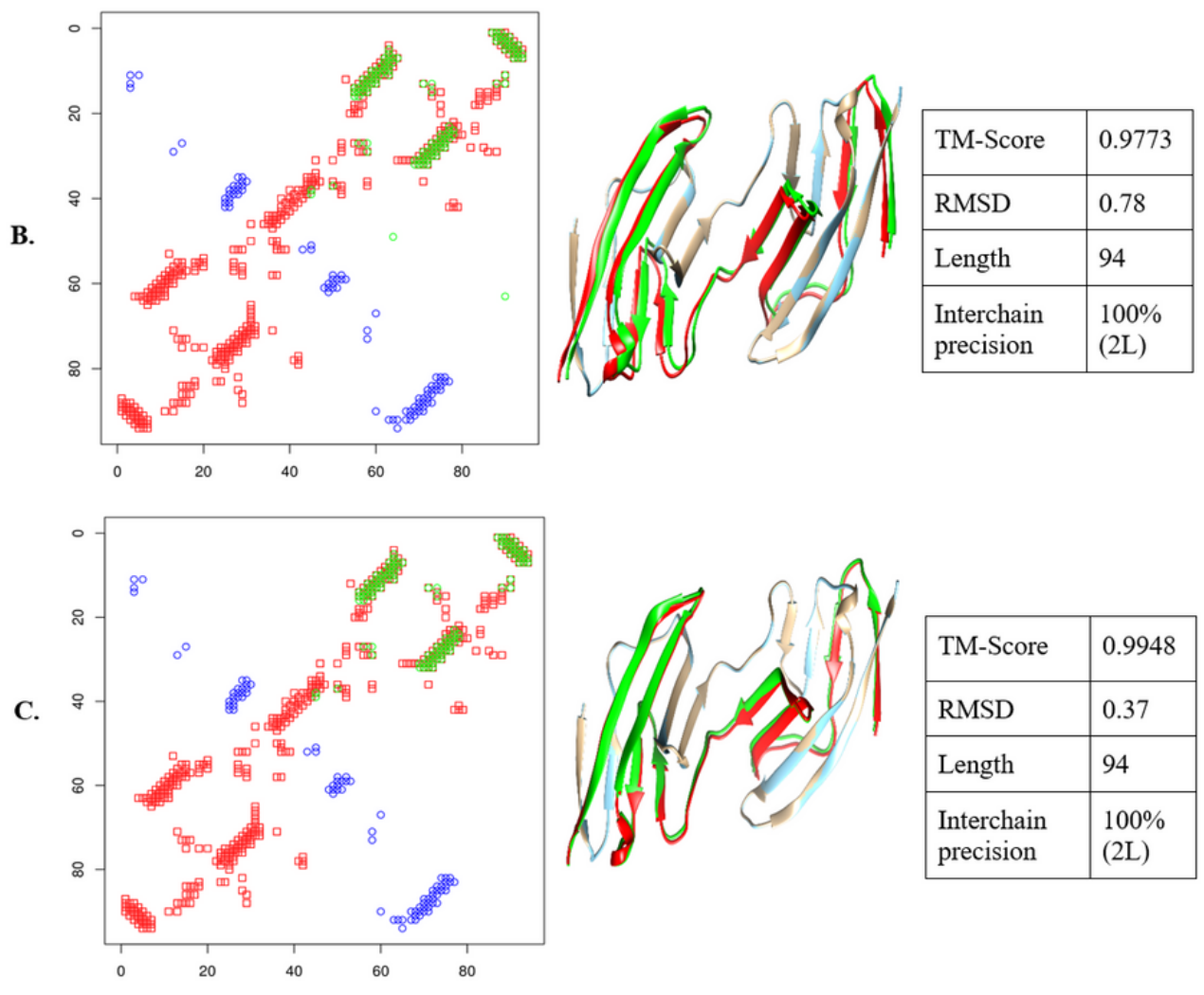

\begin{tabular}{|l|l|}
\hline TM-Score & 0.8346 \\
\hline RMSD & 2.27 \\
\hline Length & 94 \\
\hline $\begin{array}{l}\text { Interchain } \\
\text { precision }\end{array}$ & $\begin{array}{l}100 \% \\
(2 \mathrm{~L})\end{array}$ \\
\hline
\end{tabular}

$$
\text { - }
$$




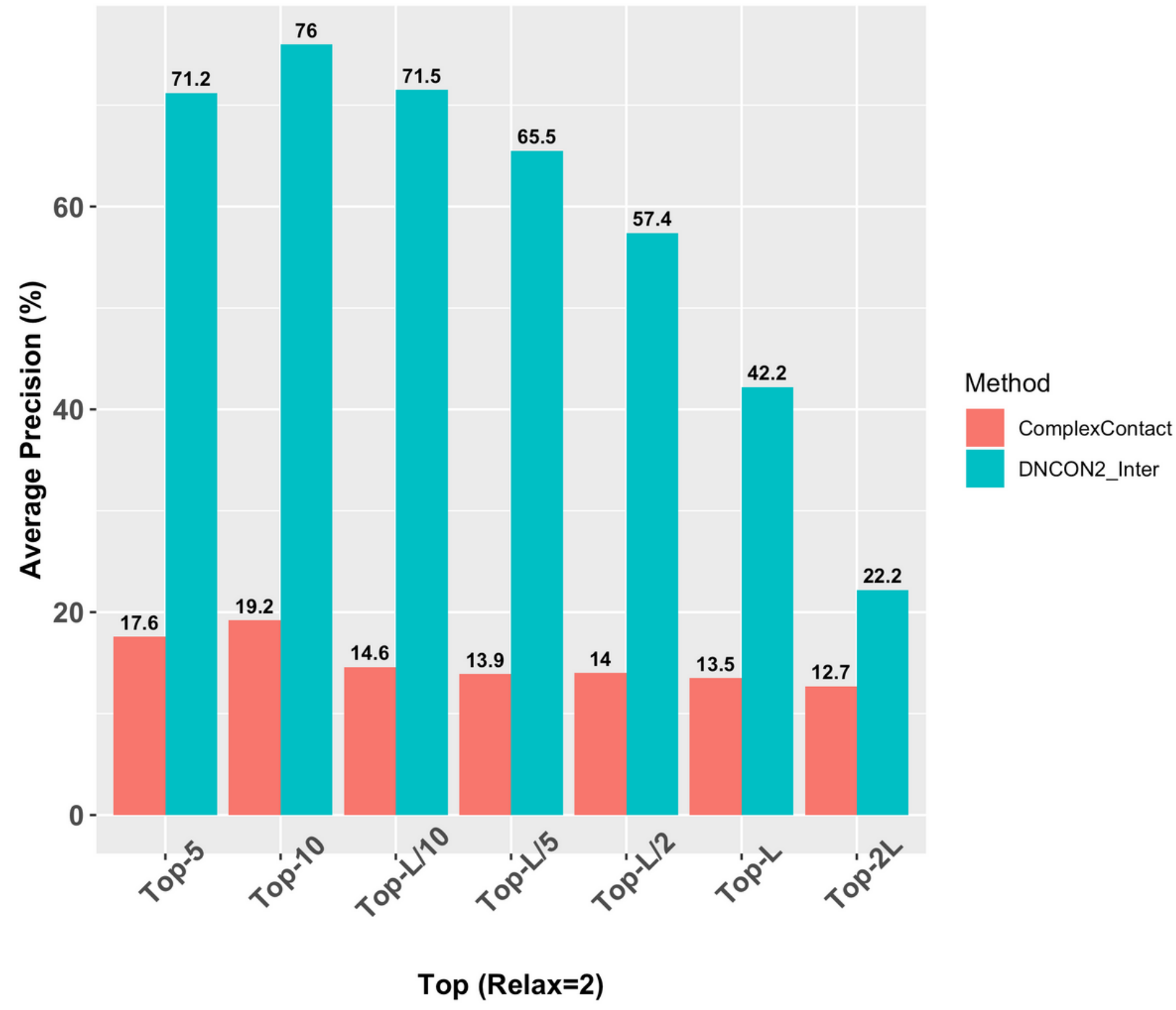

Figure 6

Shows the comparison of the average precision between ComplexContact and DNCON2_Inter on 25 randomly selected homodimers at relaxation $=2$. The best precision for both predictors is obtained for Top-10 where DNCON2_Inter outperforms ComplexContact by almost four times.

\section{Supplementary Files}

This is a list of supplementary files associated with this preprint. Click to download.

- DeepComplexDNCON2InterSupplementarySectionScienteficReports.docx 